\title{
Numerical simulation of an adaptive optics system with laser propagation in the atmosphere
}

\author{
Hai-Xing Yan, Shu-Shan Li, De-Liang Zhang, and She Chen
}

\begin{abstract}
A comprehensive model of laser propagation in the atmosphere with a complete adaptive optics (AO) system for phase compensation is presented, and a corresponding computer program is compiled. A direct wave-front gradient control method is used to reconstruct the wave-front phase. With the longexposure Strehl ratio as the evaluation parameter, a numerical simulation of an AO system in a stationary state with the atmospheric propagation of a laser beam was conducted. It was found that for certain conditions the phase screen that describes turbulence in the atmosphere might not be isotropic. Numerical experiments show that the computational results in imaging of lenses by means of the fast Fourier transform (FFT) method agree well with those computed by means of an integration method. However, the computer time required for the FFT method is 1 order of magnitude less than that of the integration method. Phase tailoring of the calculated phase is presented as a means to solve the problem that variance of the calculated residual phase does not correspond to the correction effectiveness of an $\mathrm{AO}$ system. It is found for the first time to our knowledge that for a constant delay time of an AO system, when the lateral wind speed exceeds a threshold, the compensation effectiveness of an AO system is better than that of complete phase conjugation. This finding indicates that the better compensation capability of an AO system does not mean better correction effectiveness. (C) 2000 Optical Society of America

OCIS code: $\quad 010.0010,010.1080,010.1300,010.3310,350.1260,350.4600$.
\end{abstract}

\section{Introduction}

It is well known that a turbulent atmosphere deteriorates optical waves that propagate in the atmosphere. Astronomical observation and laser propagation are the most important fields impacted by this problem. An adaptive optics (AO) system can be used to overcome, at least partially, the atmospheric deterioration of the optical wave. ${ }^{1}$ Generally, an AO system can be divided into three parts: wave-front detection, wavefront reconstruction, and wave-front correction. A Hartmann-Shack (HS) detector is used for real-time detection of the deformed wave front. The widely used wave-front reconstruction methods include the direct wave-front gradient control method ${ }^{2,3}$ and the modal wave-front reconstruction method in terms of Zernike polynomials expansion. ${ }^{4}$ A deformable mirror is often used to correct the wave front.

The authors are with the Institute of Mechanics, Chinese Academy of Sciences, Beijing 100080, China. The e-mail address for H. X. Yan is hxyan@imech.ac.cn.

Received 23 July 1999; revised manuscript received 6 December 1999.

0003-6935/00/183023-09\$15.00/0

(C) 2000 Optical Society of America
A theoretical model and numerical calculations can be used to simulate the propagation of an optical wave in a turbulent atmosphere and in the operation of an $\mathrm{AO}$ system. In this way one can design a new AO system, estimate its performance, and compare the different schemes to optimize results. Furthermore, one can perform numerical experiments for an existing AO system to investigate its operation and performance. It is possible and convenient for the numerical simulation to treat a number of different conditions, some of which might be difficult or even impossible to realize in a real experiment.

To simulate optical wave propagation in a turbulent atmosphere, Fleck and colleagues used the multiple phase-screen method to compute threedimensional laser beam propagation. ${ }^{5}$ Martin and Flatte used a similar approach to investigate the intensity and statistics by means of numerical simulation of wave propagation in three-dimensional random media. ${ }^{6}$

In theoretical studies of $\mathrm{AO}$ systems most investigators use analyses to simulate an $\mathrm{AO}$ system and calculate its performance. A number of studies have been published on this topic (see, for example, Refs. 7 and 8). A few investigators have employed a numerical simulation to study the performance of an AO 
system. Some studies have appeared in conference proceedings (see, for example, Ref. 9). However, a detailed description of the theoretical model and computational results were not included in these numerical simulation studies (see, for example, Refs. 10 and 11).

The current authors have been pursuing simultaneous numerical simulations of the atmospheric propagation of optical waves and an $\mathrm{AO}$ system since 1988. This is our first opportunity to communicate our results to our colleagues outside of China. A comprehensive theoretical model was developed and coded into a numerical algorithm. The atmospheric propagation of optical waves and the operation of an AO system were included. A laser beam propagating in the atmosphere with an active AO system was chosen as an example. Simulating the operation of a real AO system, a laser beam from a beacon propagates through a turbulent medium to obtain the deformed wave front; then the deformed wave front is detected, reconstructed, and corrected by the AO system. Finally, a phase-compensated beam from the main laser propagates in the same, but transported (because of the time delay in the AO system and the lateral wind and/or lateral movements of target and main laser), turbulent medium again to reach the target. A long-exposure Strehl ratio is used to evaluate the performance of the $\mathrm{AO}$ system.

In this paper we discuss the following: (i) A purely numerical simulation of the propagation of optical waves in a turbulent medium and in the operation of an AO system is described. (ii) The numerical simulation of the propagation of the optical wave in a turbulent medium and the numerical simulation of all portions of an AO system are combined. The turbulence-deformed wave-front phase is used as the object of treatment of the AO system, and the optical wave with compensated wave-front phase propagates in the same turbulent medium. (iii) Various conditions in the practical application of wave propagation and the $\mathrm{AO}$ system can be included in an analogous way.

\section{Theoretical Model and Numerical Simulation of Laser Propagation in a Turbulent Medium}

For optical wave propagation to the forward $(z)$ direction, Maxwell's wave equation in the parabolic or in the Fresnel approximation can be written as

$$
2 i k \frac{\partial \phi}{\partial z}+\frac{\partial^{2} \phi}{\partial x^{2}}+\frac{\partial^{2} \phi}{\partial y^{2}}+k^{2}\left(n^{2}-n_{0}^{2}\right) \phi=0
$$

where

$$
\phi(x, y, z)=E(x, y, z) \exp (-i k z) .
$$

$E(x, y, z)$ is a slowly varying wave field amplitude; the wave number $k=2 \pi / \lambda ; n$ is the refractive index; $n_{0}$ is the refractive index in the medium without turbulence; and for atmospheric propagation $n_{0}$ can be taken as 1 and

$$
n^{2}-n_{0}{ }^{2}=\left(n_{0}-n_{1}\right)^{2}-n_{0}{ }^{2} \approx 2 n_{1},
$$

where $n_{1}=n-n_{0}$ is the deviation of the refractive index, which is a stochastic quantity.

In the case of laser beam propagation a focused beam is quite important and common. For the focused beam the following coordinate transformations are used,

$$
\begin{aligned}
\bar{x} & =x /[a(1-z / l)], \\
\bar{y} & =y /[a(1-z / l)], \\
\zeta & =z /\left(k a^{2}\right),
\end{aligned}
$$

where $a$ is the radius of the beam and $l$ is a transformation factor; $l>0$ corresponds to a convergent beam, $l<0$ corresponds to a divergent beam, and $l \rightarrow$ $\infty$ corresponds to a parallel beam.

Propagation equation (1) becomes

$$
2 i \frac{\partial \psi}{\partial \zeta}+\frac{1}{\left(1-k a^{2} \zeta / l\right)^{2}}\left(\frac{\partial^{2} \psi}{\partial \bar{x}^{2}}+\frac{\partial^{2} \psi}{\partial \bar{y}^{2}}\right)+2 k^{2} a^{2} n_{1} \psi=0,
$$

where

$$
\begin{aligned}
\psi(\bar{x}, \bar{y}, \zeta)= & \left(\frac{c a^{2}}{8 \pi P_{T}}\right)^{1 / 2} \\
& \times(1-z / l) \phi(x, y, z) \exp \left[\frac{i k\left(x^{2}+y^{2}\right)}{2 l(1-z / l)}\right],
\end{aligned}
$$

$P_{T}$ is the total beam power, and $c$ is the speed of light. Equation (5) is the basic propagation equation used in the numerical simulation. It is changed into a difference equation and solved numerically.

When we adopt an approach similar to that of Fleck et $a l .,{ }^{5}$ the multiple phase-screen model is used to describe the turbulent medium. The main idea is as follows. The effect of turbulence on the optical wave is expressed in terms of random change in the refractive index $n_{1}$. The propagation path through the turbulent medium can be divided into several segments, which may have different lengths. In this study equally spaced segments are used for simplicity. It is thought that each segment may deform the phase of the optical wave independently but does not have a significant influence on the amplitude of the wave. The amplitude of the optical wave is changed only in the propagation process of the wave with the deformed phase. Changes in amplitude and phase of the optical wave can be treated separately. Then the contribution of the turbulent medium segment to the phase of the optical wave can be pressed into a thin phase screen and added to the initial phase of the wave. The wave with changed phase then propagates to the next phase screen in a medium without turbulence. At the position of the next phase screen, the wave with changed amplitude is modified by the new phase screen and so on. This process continues until the wave reaches the target.

The effect of a turbulent medium segment on the optical wave phase can be deduced as follows. In 
propagation equation (5), remove the terms for diffraction, and get

$$
2 i \frac{\partial \psi}{\partial \zeta}+2 k^{2} a^{2} n_{1} \psi=0 .
$$

The solution is

$$
\begin{aligned}
\psi(\bar{x}, \bar{y}, \zeta+\Delta \zeta) & =\psi(\bar{x}, \bar{y}, \zeta) \exp \left(i k^{2} a^{2} n_{1} \Delta \zeta\right) \\
& =\psi(\bar{x}, \bar{y}, \zeta) \exp \left(i k n_{1} \Delta z\right)
\end{aligned}
$$

where $\Delta z=k a^{2} \Delta \zeta$ is the step length in the $z$ direction. The effect of a turbulent medium segment is equivalent to adding an additional phase $n_{1} \Delta z$ to the initial phase of the optical wave. In a practical calculation it is assumed that the additional phase is added in an infinitesimally thin phase screen, i.e.,

$$
\psi\left(\bar{x}, \bar{y}, \zeta^{+}\right)=\psi(\bar{x}, \bar{y}, \zeta) \exp \left(i k n_{1} \Delta z\right) .
$$

Then the wave field $\psi\left(\bar{x}, \bar{y}, \zeta^{+}\right)$propagates to reach the next phase screen in a medium without turbulence to obtain $\psi(\bar{x}, \bar{y}, \zeta+\Delta \zeta)$. Now the problem is to determine the additional phase change $\Gamma(x, y)$ resulting from a turbulent medium segment $\Delta z$.

It can be proved that in the difference form

$$
\begin{aligned}
\Gamma(x, y)= & n_{1} \Delta z \\
= & \left(\pi \Delta z \Delta k_{x} \Delta k_{y}\right)^{1 / 2} \sum_{I=-\left(N_{x} / 2\right)+1}^{N_{x} / 2} \sum_{J=-\left(N_{y} / 2\right)+1}^{N_{y} / 2} \exp \left(i I \Delta k_{x} x\right. \\
& \left.+i J \Delta k_{y} y\right) \Phi_{n}{ }^{1 / 2}\left(I \Delta k_{x}, J \Delta k_{y}\right)\left[a_{1}\left(I \Delta k_{x}, J \Delta k_{y}\right)\right. \\
& \left.+i a_{2}\left(I \Delta k_{x}, J \Delta k_{y}\right)\right],
\end{aligned}
$$

where $\Phi_{n}\left(k_{x}, k_{y}\right)=\Phi_{n}\left(k_{x}, k_{y}, 0\right)$ is the spectral density for the refractive-index fluctuations. Assuming a von Karman spectrum,

$$
\Phi_{n}\left(k_{x}, k_{y}\right)=0.033 C_{n}^{2}\left(k_{0}{ }^{2}+k_{x}{ }^{2}+k_{y}{ }^{2}\right)^{-11 / 6} .
$$

Here $C_{n}{ }^{2}$ is the index structure constant and $k_{0}=$ $2 \pi / L_{0}$, where $L_{0}$ is the outer scale length of the turbulence. The formula deduction requires that $\Delta z \gg$ $L_{0} . \quad a_{1}$ and $a_{2}$ are two-dimensional complex random numbers. These independent random numbers are assumed to obey Gaussian statistics and to have variance 1. Furthermore, it is required that

$$
\begin{aligned}
& a_{1}\left(k_{x}, k_{y}\right)=a_{1}\left(-k_{x},-k_{y}\right), \\
& a_{2}\left(k_{x}, k_{y}\right)=-a_{2}\left(-k_{x},-k_{y}\right) .
\end{aligned}
$$

The step lengths $\Delta k_{x}$ and $\Delta k_{y}$ for wave numbers $k_{x}$ and $k_{y}$ are

$$
\begin{aligned}
& \Delta k_{x}=\frac{2 \pi}{N_{x} \Delta x}=\frac{2 \pi}{N_{x} \Delta \bar{x} a\left(1-k a^{2} \zeta / l\right)}, \\
& \Delta k_{y}=\frac{2 \pi}{N_{y} \Delta y}=\frac{2 \pi}{N_{y} \Delta \bar{y} a\left(1-k a^{2} \zeta / l\right)},
\end{aligned}
$$

where $N_{x}, N_{y}$ are the grid numbers in the $x$ and the $y$ directions and $\Delta x, \Delta y$ are the corresponding step lengths.

When we combine Eqs. (10)-(13), $\Gamma(x, y)$ can be

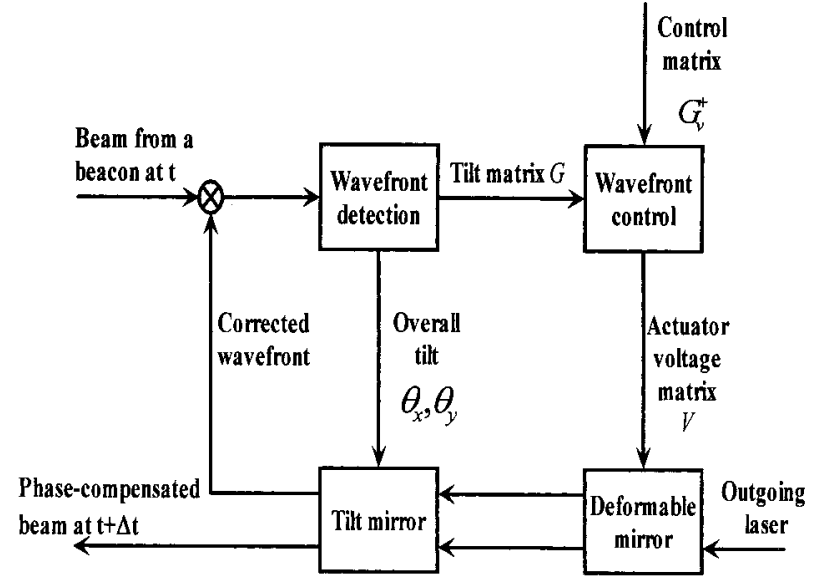

Fig. 1. Combination of numerical simulation of laser propagation in the atmosphere with that of an AO system.

calculated from the double sum (assuming the grid numbers $N_{x}=N_{y}=N$ ),

$$
\begin{aligned}
& \Gamma\left(m_{x} \Delta x, m_{y} \Delta y\right)=\frac{2 \pi}{N}\left(\frac{0.033 \pi \Delta z C_{n}^{2}}{\Delta x \Delta y}\right)^{1 / 2} \\
& \times \sum_{I=-(N / 2)+1} \sum_{J=-(N / 2)+1}^{N / 2} \frac{\exp \left(\frac{2 \pi \operatorname{Iim}_{x}}{N}+\frac{2 \pi J i m_{y}}{N}\right)}{\left[\left(\frac{2 \pi}{L_{0}}\right)^{2}+\left(\frac{2 \pi I}{N \Delta x}\right)^{2}+\left(\frac{2 \pi J}{N \Delta y}\right)^{2}\right]^{11 / 12}} \\
& \times\left[a_{1}\left(I \Delta k_{x}, J \Delta k_{y}\right)+i a_{2}\left(I \Delta k_{x}, J \Delta k_{y}\right)\right],
\end{aligned}
$$

which can be computed by a sequence of fast Fourier transform (FFT) operations.

\section{Theoretical Model and Numerical Simulation of an Adaptive Optics System}

The research presented here includes only the simulation of an $\mathrm{AO}$ system in a stationary state. In a practical AO system, the dynamic control process must be included to make the system stable. The dynamic control process is ignored here. It is well known that an $\mathrm{AO}$ system must have a time delay, because the system has a limited response and the wave-front detection, reconstruction, and correction take some time. The effects of the time delay are treated as follows. On the basis of the well-known Taylor hypothesis, the turbulence (i.e., the phase screens) is considered to be frozen in a short time period (for example, for a few milliseconds). The phase screens are assumed to move laterally in the delay time period because of lateral wind and/or the lateral movements of the laser and target. Thus the laser beam with compensated phase will propagate through the laterally moved phase screens to reach the target (see Fig. 1). The phase reconstruction used here is based on the direct wave-front gradient control method. ${ }^{2,3}$ The effects of noise and detection errors in the $\mathrm{AO}$ system are ignored here. 


\section{A. Wave-Front Detection}

The wave field of the beam that radiates from a beacon propagates through a turbulent medium and arrives at a HS detector. The detector is divided into several subapertures. The wave field of a subaperture $u_{1}\left(x_{0}, y_{0}\right)$ propagates to reach the focal plane of the HS detector. Its wave field becomes

$$
u_{2}(x, y)=\iint_{\Sigma} \frac{f}{i \lambda r^{2}} \exp (i k r) u_{1}\left(x_{0}, y_{0}\right) \mathrm{d} x_{0} \mathrm{~d} y_{0}
$$

where $\iint_{\Sigma}$ expresses the integral over the subaperture and $r$ is the distance between points $(x, y)$ on the focal plane and $\left(x_{0}, y_{0}\right)$ on the subaperture. The position of the optical center can be calculated as

$$
\begin{aligned}
& x_{c}=\iint_{T} x\left|u_{2}(x, y)\right|^{2} \mathrm{~d} x \mathrm{~d} y / \iint_{T}\left|u_{2}(x, y)\right|^{2} \mathrm{~d} x \mathrm{~d} y, \\
& y_{c}=\iint_{T} y\left|u_{2}(x, y)\right|^{2} \mathrm{~d} x \mathrm{~d} y / \iint_{T}\left|u_{2}(x, y)\right|^{2} \mathrm{~d} x \mathrm{~d} y,
\end{aligned}
$$

where $\iint_{T}$ expresses the integral over the focal plane. When we take the position of the optical center of the focused plane-wave field of the subaperture as 0 , the average tilts of the subaperture in the $x$ and the $y$ directions can be calculated as

$$
\begin{aligned}
& G_{x}=(2 \pi / f \lambda) x_{c}, \\
& G_{y}=(2 \pi / f \lambda) y_{c}
\end{aligned}
$$

where $f$ is the focal length of the subaperture.

In a practical $\mathrm{AO}$ system the overall tilt of the wave front is corrected by a specific tilt-correction mirror. In the numerical simulation the overall tilts in the $x$ and the $y$ directions are separated as

$$
\begin{aligned}
& \theta_{x}=\frac{1}{m} \sum_{i=1}^{m} G_{x}(i), \\
& \theta_{y}=\frac{1}{m} \sum_{i=1}^{m} G_{y}(i),
\end{aligned}
$$

where $m$ is the number of the subaperture and $G_{x}(i)$ and $G_{y}(i)$ are the average tilts of the $i$ th subaperture in the $x$ and the $y$ directions, respectively. The average tilts of each subaperture after subtraction of the overall tilts in the $x$ and the $y$ directions constitute the tilt matrix $G$. In this paper it is assumed that the tilt-correction mirror can completely correct the overall tilts $\theta_{x}$ and $\theta_{y}$.

\section{B. Wave-Front Reconstruction}

The main point of wave-front reconstruction is to obtain the control matrix. The control matrix multiplies the tilt matrix $G$ to get the actuator voltage matrix $V$. The control matrix is determined by use of the direct wave-front gradient control method.
The surface shape of a deformable mirror that is the corrected wave front can be expressed as

$$
\psi_{m}(x, y)=\sum_{j=1}^{K} V_{j} R_{j}(x, y),
$$

where $K$ is the total number of actuators on the deformable mirror, $V_{j}$ is the voltage of the $j$ th actuator, and $R_{j}(x, y)$ is the influence function of the $j$ th actuator, which describes the effect of a unit displacement of the $j$ th actuator on the surface shape of the deformable mirror. In principle, the influence function of each actuator may be different, and this effect can be included in the numerical simulation. For this study it is assumed that a Gaussian influence function can be used for all actuators of the deformable mirror,

$$
R_{j}(x, y)=\frac{2 \pi}{\lambda} \exp \left\{\ln b\left[\left(x-x_{j}\right)^{2}+\left(y-y_{j}\right)^{2}\right] / d^{2}\right\},
$$

where $b$ is the coupling factor between the adjacent actuators, $x_{j}$ and $y_{j}$ are the coordinates of the $j$ th actuator, and $d$ is the distance between the adjacent actuators. In fact, any type of influence function for the actuator can be used in the simulation computation.

Differentiating the corrected wave front $\psi_{m}(x, y)$ with respect to $x$ and $y$ and averaging over the subaperture, we can show that

$$
\begin{aligned}
& G_{x i}=\sum_{j=1}^{K} G_{v x i j} V_{j}, \\
& G_{y i}=\sum_{j=1}^{K} G_{v y i j} V_{j},
\end{aligned}
$$

where

$$
\begin{aligned}
G_{v x i j} & =\frac{1}{s_{i}} \iint_{S_{i}} \frac{\partial R_{j}(x, y)}{\partial x} \mathrm{~d} x \mathrm{~d} y, \\
G_{v y i j} & =\frac{1}{s_{i}} \iint_{S_{i}} \frac{\partial R_{j}(x, y)}{\partial y} \mathrm{~d} x \mathrm{~d} y .
\end{aligned}
$$

Here $\iint_{S_{i}}$ is the integral on the $i$ th subaperture and $S_{i}$ is the area of the $i$ th subaperture. $\quad G_{v x i j}$ and $G_{v y i j}$ can be considered to be the average tilts on the $i$ th subaperture produced by a unit displacement of the $j$ th actuator. In matrix form,

$$
G=G_{v} V
$$

Equation (28) can be solved with the least-squares method to obtain

$$
V=G_{v}^{+} G
$$

where $G_{v}^{+}$is the generalized inverse matrix with least-squares and minimum norm of matrix $G_{v} . G_{v}^{+}$ can be deduced by decomposition of singular values. $G_{v}^{+}$is the control matrix of the direct gradient control wave-front reconstruction. 
Table 1. Six Strehl Ratios

\begin{tabular}{ccccccccc}
\hline Times & 1 & \multicolumn{1}{c}{2} & \multicolumn{1}{c}{3} & \multicolumn{1}{c}{30} & \multicolumn{1}{c}{40} & 50 & 100 & 200 \\
\hline STRA & 0.0675 & 0.0480 & 0.0409 & 0.0158 & 0.0145 & 0.0139 & 0.0137 \\
STRB & 0.0154 & 0.0109 & 0.0113 & 0.0089 & 0.0112 & 0.0116 & 0.0119 & 0.0132 \\
STRC & 0.0188 & 0.0062 & 0.0139 & 0.0089 & 0.0112 & 0.0116 & 0.0121 \\
STRAA & 0.254 & 0.200 & 0.210 & 0.143 & 0.156 & 0.157 & 0.162 \\
STRBB & 0.132 & 0.121 & 0.170 & 0.155 & 0.162 & 0.161 & 0.164 \\
STRCC & 0.225 & 0.197 & 0.192 & 0.155 & 0.162 & 0.161 & 0.164 \\
\hline
\end{tabular}

Computational conditions: $\lambda=0.6328 \mu \mathrm{m} ; L=1 \mathrm{~km}$; open loop; $C_{n}{ }^{2}=10^{-14} \mathrm{~m}^{-2 / 3}$; phase-screen grids, $512 \times 512$; propagation grids, $128 \times 128$, in center; ten phase screens.

Table 2. STRCC in Different Turbulent Media

\begin{tabular}{|c|c|c|c|c|c|c|c|c|c|}
\hline$C_{n}{ }^{2}\left(\mathrm{~m}^{-2 / 3}\right)$ & Times & 1 & 2 & 3 & 30 & 40 & 50 & 100 & 200 \\
\hline \multirow{2}{*}{$10^{-14.5}$} & $\mathrm{OL}$ & 0.596 & 0.551 & 0.492 & 0.496 & 0.520 & 0.514 & 0.495 & 0.502 \\
\hline & $\mathrm{AO}$ & 0.881 & 0.883 & 0.876 & 0.859 & 0.858 & 0.858 & 0.856 & 0.854 \\
\hline \multirow{2}{*}{$10^{-14}$} & $\mathrm{OL}$ & 0.225 & 0.197 & 0.192 & 0.155 & 0.162 & 0.161 & 0.164 & 0.164 \\
\hline & $\mathrm{AO}$ & 0.649 & 0.601 & 0.585 & 0.605 & 0.612 & 0.616 & 0.614 & 0.612 \\
\hline \multirow{2}{*}{$10^{-13.5}$} & $\mathrm{OL}$ & 0.0978 & 0.0634 & 0.0613 & 0.0536 & 0.0532 & 0.0523 & 0.0487 & 0.0490 \\
\hline & $\mathrm{AO}$ & 0.165 & 0.214 & 0.210 & 0.223 & 0.221 & 0.220 & 0.214 & 0.212 \\
\hline
\end{tabular}

Computational conditions: 61-unit AO system; others, see Table 1. OL, open loop.

Table 3. Effect of Grid Number of Propagation on Strehl Ratio

\begin{tabular}{|c|c|c|c|c|c|c|}
\hline \multirow{2}{*}{$\frac{C_{n}^{2}\left(\mathrm{~m}^{-2 / 3}\right)}{\text { Grid number }}$} & \multicolumn{2}{|c|}{$10^{-13.5}$} & \multicolumn{2}{|c|}{$10^{-14}$} & \multicolumn{2}{|c|}{$10^{-14.5}$} \\
\hline & $128 \times 128$ & $256 \times 256$ & $128 \times 128$ & $256 \times 256$ & $128 \times 128$ & $256 \times 256$ \\
\hline STRCC & 0.0523 & 0.0538 & 0.161 & 0.193 & 0.514 & 0.524 \\
\hline
\end{tabular}

Computational conditions: 50 turbulence realizations, open loop, propagation grids in center; others, see Table 1.

\section{Wave-Front Correction}

In a practical AO system the wave-front correction is realized by a high speed tilt-correction mirror and a deformable mirror. Correspondingly, the corrected wave-front phase of the main laser beam can be expressed as

$$
\psi_{c}=\psi_{\mathrm{tilt}}+\psi_{m}=\theta_{x} x+\theta_{y} y+\sum_{j=1}^{K} V_{j} R_{j}(x, y) .
$$

\section{Results and Discussion}

The Strehl ratio is a useful parameter for describing the propagation quality of an optical wave. Six Strehl ratios are used in the numerical simulation. The ratio of the brightness of the brightest point on the target after propagation through the turbulent medium and through a vacuum is defined as STRA. The ratio of the optical energies within a circle around the brightest point with a radius of the first dark ring in the Airy pattern after propagation through the turbulent medium and through a vacuum is defined as STRAA. STRB and STRBB are similar ratios for the center point on the target instead of the brightest point. STRC and STRCC are the ratios for the optical center on the target. The long-exposure Strehl ratio obtained from the accumulated wave field is used, since it is similar to experimental observations.

Table 1 shows that it is necessary to average over many turbulence realizations to get relatively stable results. Fifty realizations appear to be sufficient. Also note that the values for averaged STRA, STRB, and STRC are comparable, the averaged STRAA, STRBB, and STRCC are comparable, and the former values are much smaller than the latter values.

The relative variances of STRA, STRB, and STRC are always larger than those of STRAA, STRBB, and STRCC. In other words, fluctuations of STRA, STRB, and STRC are larger than those of STRAA, STRBB, and STRCC. We chose STRCC as the evaluation parameter.

It is shown in Table 2 that in turbulent media with different strengths, 40-50 realizations generally give a relatively stable averaged Strehl ratio, and the Strehl ratio rapidly becomes worse as the turbulence grows stronger.

Table 4. Test of Isotropism of Phase Screens (I)

\begin{tabular}{lrrccc}
\hline Position & \multicolumn{1}{c}{$x$ Grid } & \multicolumn{1}{c}{$y$ Grid } & 10 Times & 20 Times & 50 Times \\
\hline Left 1 & $65-192$ & $193-320$ & 0.276 & 0.285 & 0.262 \\
Center & $193-320$ & $193-320$ & 0.229 & 0.214 & 0.238 \\
Right 1 & $321-448$ & $193-320$ & 0.230 & 0.181 & 0.228 \\
Upper 1 & $193-320$ & $321-448$ & 0.315 & 0.372 & 0.362 \\
Center & $193-320$ & $193-320$ & 0.229 & 0.214 & 0.238 \\
Lower 1 & $193-320$ & $65-192$ & 0.238 & 0.210 & 0.181 \\
\hline
\end{tabular}

Computational conditions: $\lambda=0.6328 \mu \mathrm{m} ; L=340 \mathrm{~m}$; open loop, $C_{n}{ }^{2}=2.78 \times 10^{-14} \mathrm{~m}^{-2 / 3}\left(r_{0}=5 \mathrm{~cm}\right)$; phase screens of the first kind; phase screen grids, $512 \times 512$; propagation grids, $128 \times$ 128; ten phase screens. 
Table 5. Test of Isotropism of Phase Screens (II)

\begin{tabular}{lrrccccc}
\hline Position & $x$ Grid & $y$ Grid & 10 Times & 20 Times & 50 Times & 100 Times & 200 Times \\
\hline Left 1 & $65-192$ & $193-320$ & 0.157 & 0.180 & 0.183 & 0.170 & 0.169 \\
Center & $193-320$ & $193-320$ & 0.161 & 0.166 & 0.161 & 0.164 & 0.164 \\
Right 1 & $321-448$ & $193-320$ & 0.155 & 0.165 & 0.181 & 0.174 & 0.165 \\
Upper 1 & $193-320$ & $321-448$ & 0.205 & 0.168 & 0.161 & 0.164 & 0.164 \\
Center & $193-320$ & $193-320$ & 0.161 & 0.166 & 0.161 & 0.179 \\
Lower 1 & $193-320$ & $65-192$ & 0.216 & 0.198 & 0.178 & 0.167 \\
\hline
\end{tabular}

Computational conditions: same as Table 1 except for the propagation grid position, phase screen of the second kind.

Table 3 shows that computational results with a $128 \times 128$ grid are comparable with those with a $256 \times 256$ grid. The computer time required with the $128 \times 128$ grid is approximately one fourth of that with a $256 \times 256$ grid.

An improper phase-screen creation method, i.e., an improper random-number generator, can give rise to phase screens without isotropism (see Table 4). The averaged Strehl ratios after passing through the phase screens of the first kind at different positions in the $x$ direction are comparable. However, that is not the case in the $y$ direction. We checked independent random numbers $a_{1}\left(k_{x}, k_{y}\right)$ and $a_{2}\left(k_{x}, k_{y}\right)$ [see Eqs. (10) and (14)] carefully and found that they obey Gaussian statistics and have variance 1 . With another kind of random numbers, a second kind of phase screen was produced that is perfectly isotropic (see Table 5).

It was found that, in contrast to Ref. 5 , the coordinate transformations, Eqs. (4), for a focused beam are profitable in that the positions of the phase screens are not influenced by the coordinate transformations. It is quite easy to arrange equally spaced screens. Another advantage of the coordinate transformations is that it is possible to get comparable results by use of fewer phase screens, so computer time can be reduced (see Table 6).

The beam from a beacon may propagate in a strong turbulent medium to reach the HS detector. Figures 2 and 3 show a disturbed phase wave front, which is calculated from our numerical simulation for this case. Figure 4 shows the corrected phase after the correction of an AO system of 61 units (with 48 subapertures and 61 actuators). The correspondence of Figs. 2 and 4 is quite poor, although the Strehl ratio $(\mathrm{STRCC}=0.673)$ shows that the phase

Table 6. STRCC in the Different Turbulent Media

\begin{tabular}{cccccccccc}
\hline & & \multicolumn{3}{c}{ 10 Phase Screens } & & \multicolumn{3}{c}{ 20 Phase Screens } \\
\cline { 8 - 9 } \cline { 7 - 9 } $\begin{array}{c}C_{n}{ }^{2} \\
\left(\mathrm{~m}^{-2 / 3}\right)\end{array}$ & Times & 50 & 100 & 200 & & 50 & 100 & 200 \\
\hline \multirow{2}{*}{$10^{-14.5}$} & OL & 0.490 & 0.481 & 0.475 & & 0.482 & 0.477 & 0.462 \\
& AO & 0.855 & 0.854 & 0.853 & & 0.857 & 0.854 & 0.852 \\
$10^{-14}$ & OL & 0.161 & 0.164 & 0.164 & & 0.162 & 0.166 & 0.160 \\
& AO & 0.616 & 0.614 & 0.612 & & 0.616 & 0.611 & 0.607 \\
$10^{-13.5}$ & OL & 0.0451 & 0.0447 & 0.0448 & & 0.0469 & 0.0472 & 0.0451 \\
& AO & 0.215 & 0.211 & 0.206 & & 0.204 & 0.202 & 0.201 \\
\hline
\end{tabular}

Computational conditions: 61-unit AO system; others, see Table 1 . OL, open loop. compensation should be quite good. This apparent anomaly results because the calculated phase has discontinuities. The phase calculated from inverse trigonometric functions is limited to changes of $2 \pi$. The discontinuities in the calculated phase are artifacts of the trigonometric functions. In practice, it is impossible for the wave-front phase to have such discontinuities; even the wave front is greatly deformed

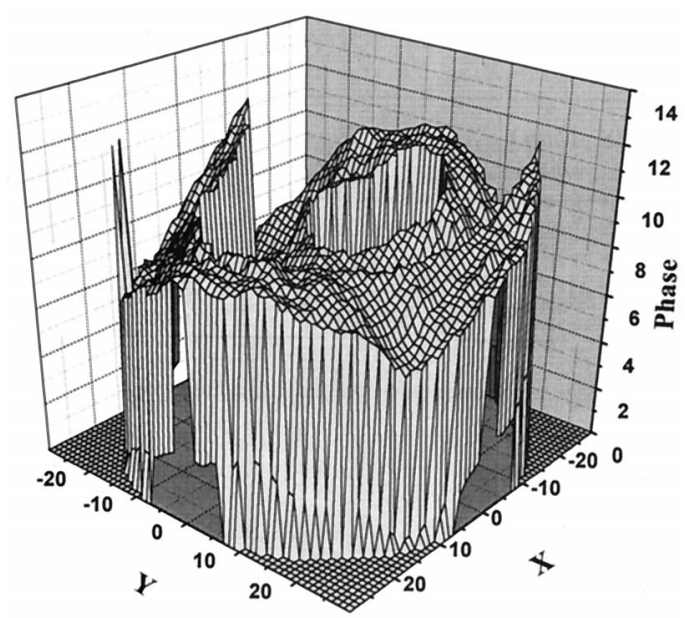

Fig. 2. Surface drawing of deformed phase wave front including overall tilt before phase tailoring.

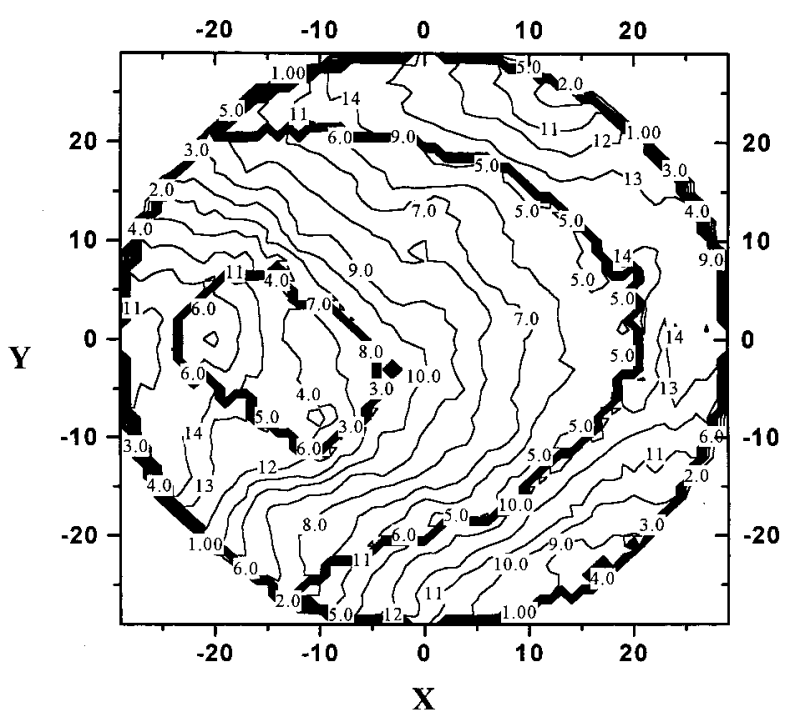

Fig. 3. Topographic drawing of phase wave front of Fig. 2. 


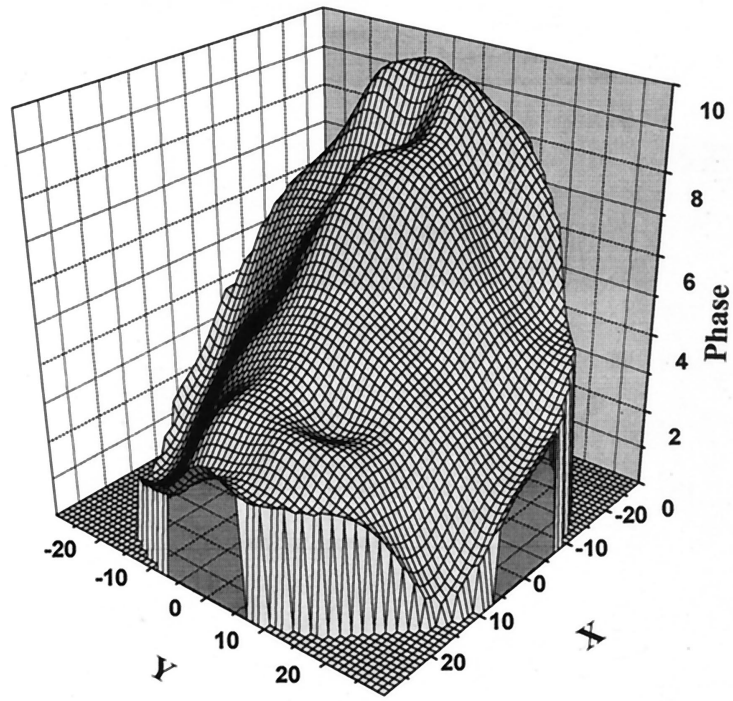

Fig. 4. Surface drawing of AO-corrected phase wave front of Fig. 2.

by the turbulent medium. We devised a technique called phase tailoring to remove the phase discontinuities. Figure 5 shows the disturbed phase of Fig. 2 after phase tailoring. The correspondence of Figs. 4 and 5 is quite good. Figure 6 shows the residual phase after $\mathrm{AO}$ phase compensation and phase tailoring. Figures 7 and 8 show a cross section of the disturbed phase of Figs. 2 and 5, respectively. It is clear that phase discontinuities are removed after phase tailoring.

For an AO system in a stationary state, in the case of strong turbulence three to four iterations are needed to get the best correction effect for one turbulence realization (see Table 7). It can be seen from Table 7 that the Strehl ratio does not correspond to phase variance before phase tailoring, but the corre-

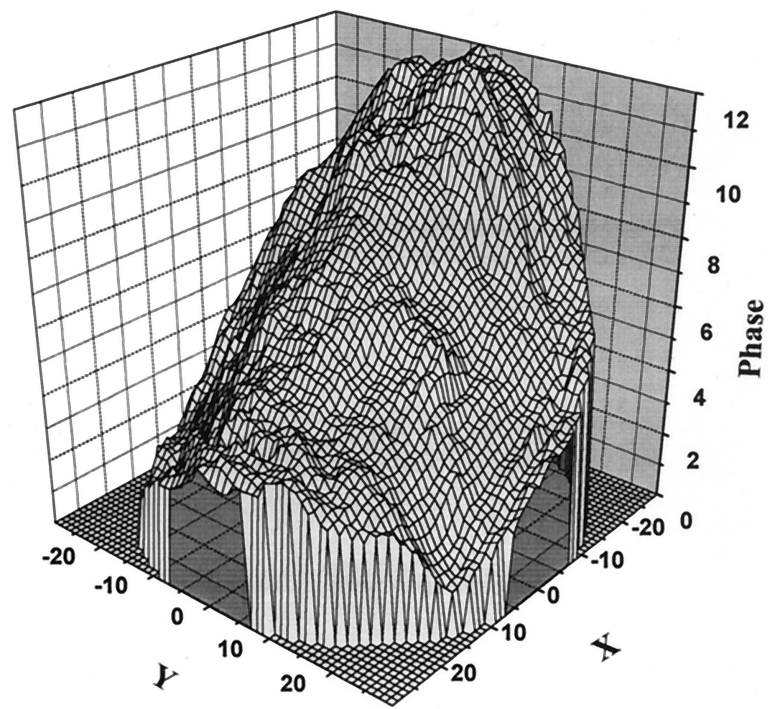

Fig. 5. Surface drawing of phase wave front of Fig. 2 after phase tailoring.

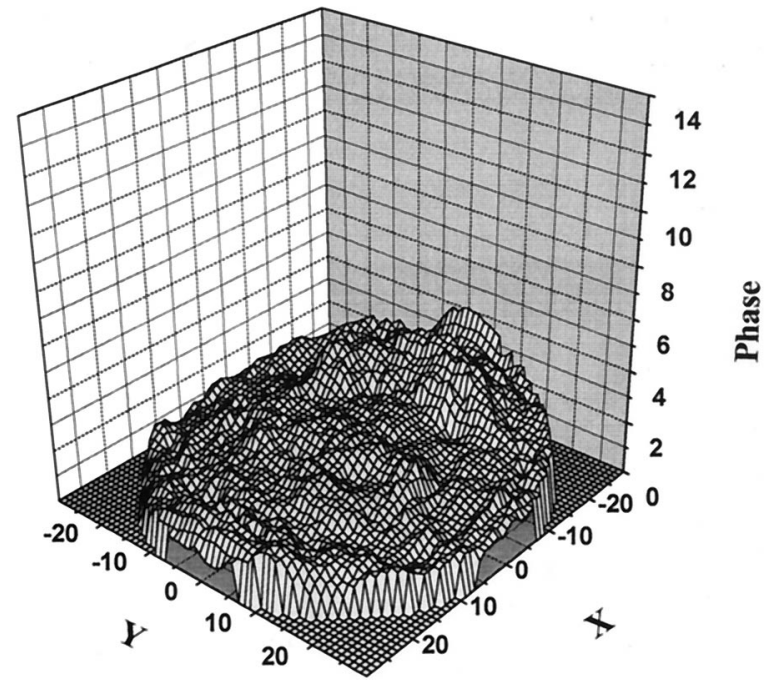

Fig. 6. Surface drawing of residual phase wave front of Fig. 2 after phase tailoring.

spondence between Strehl ratio and phase variance is quite good after phase tailoring. In the case of weak turbulence, phase tailoring is not needed, and one to two iterations are enough to obtain the best correction.

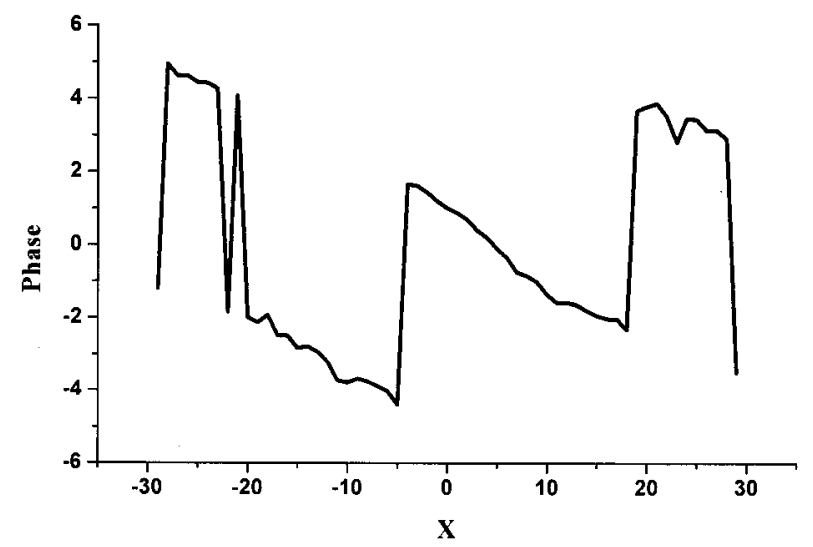

Fig. 7. Cross section of phase wave front of Fig. 2 at $y$ grid point -4 .

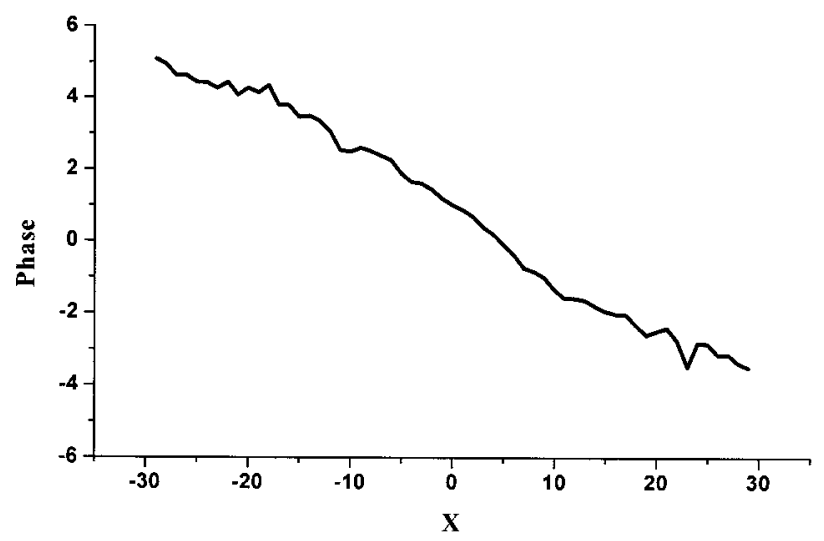

Fig. 8. Cross section of phase wave front of Fig. 5 at $y$ grid point -4 . 
Table 7. Strehl Ratio and Phase Variance $\sigma_{\varphi}{ }^{2}$ without the Overall Tilt of an AO System

\begin{tabular}{|c|c|c|c|c|c|c|c|c|c|c|}
\hline \multirow{3}{*}{$\begin{array}{c}\text { Condition } \\
\text { Iteration Times } \\
0(\mathrm{OL})\end{array}$} & \multicolumn{2}{|c|}{1} & \multicolumn{2}{|c|}{2} & \multicolumn{2}{|c|}{3} & \multicolumn{2}{|c|}{4} & \multicolumn{2}{|c|}{5} \\
\hline & STRCC & $\sigma_{\phi}^{2}$ & STRCC & $\sigma_{\phi}^{2}$ & STRCC & $\sigma_{\phi}^{2}$ & STRCC & $\sigma_{\phi}^{2}$ & STRCC & $\sigma_{\phi}^{2}$ \\
\hline & 0.469 & 4.7568 & 0.469 & 2.0776 & 0.835 & 1.7969 & 0.922 & 0.1573 & 0.338 & 2.3095 \\
\hline 1 & 0.772 & 4.8937 & 0.772 & 0.5566 & 0.954 & 0.0474 & 0.979 & 0.0200 & 0.762 & 0.3078 \\
\hline 2 & 0.810 & 5.2581 & 0.810 & 0.4695 & 0.965 & 0.0345 & 0.983 & 0.0146 & 0.818 & 0.2256 \\
\hline 3 & 0.813 & 5.4267 & 0.813 & 0.3543 & 0.965 & 0.0347 & 0.983 & 0.0147 & 0.818 & 0.2271 \\
\hline 4 & 0.815 & 5.4437 & 0.815 & 0.2975 & 0.965 & 0.0342 & 0.983 & 0.0145 & 0.819 & 0.2233 \\
\hline 5 & 0.816 & 5.4341 & 0.816 & 0.2652 & 0.965 & 0.0343 & 0.983 & 0.0145 & 0.819 & 0.2229 \\
\hline 6 & 0.815 & 5.5034 & 0.815 & 0.2486 & 0.965 & 0.0342 & 0.983 & 0.0144 & 0.819 & 0.2219 \\
\hline 7 & 0.815 & 5.4999 & 0.815 & 0.2401 & 0.965 & 0.0341 & 0.983 & 0.0144 & 0.819 & 0.2215 \\
\hline 8 & 0.814 & 5.4735 & 0.814 & 0.2359 & 0.965 & 0.0341 & 0.983 & 0.0143 & 0.819 & 0.2211 \\
\hline 9 & 0.814 & 5.4757 & 0.814 & 0.2341 & 0.965 & 0.0341 & 0.983 & 0.0144 & 0.819 & 0.2208 \\
\hline 10 & 0.814 & 5.4707 & 0.814 & 0.2334 & 0.965 & 0.0341 & 0.983 & 0.0144 & 0.819 & 0.2206 \\
\hline
\end{tabular}

Computational conditions: $\lambda=0.6328 \mu \mathrm{m} ; L=340 \mathrm{~m}$; 37-unit AO system; phase-screen grids, $512 \times 512$; propagation grids, $128 \times$ 128; ten phase screens; one turbulence realization. Condition $1: C_{n}^{2}=2.78 \times 10^{-14} \mathrm{~m}^{-2 / 3}\left(r_{0}=5 \mathrm{~cm}\right)$, before phase tailoring. Condition 2: $C_{n}{ }^{2}=2.78 \times 10^{-14} \mathrm{~m}^{-2 / 3}\left(r_{0}=5 \mathrm{~cm}\right)$, after phase tailoring. Condition 3: $C_{n}{ }^{2}=4.455 \times 10^{-15} \mathrm{~m}^{-2 / 3}\left(r_{0}=15 \mathrm{~cm}\right)$, before phase tailoring. Condition 4: $C_{n}{ }^{2}=1.90 \times 10^{-15} \mathrm{~m}^{-2 / 3}\left(r_{0}=25 \mathrm{~cm}\right)$, before phase tailoring. Condition $5: C_{n}{ }^{2}=2.78 \times 10^{-14} \mathrm{~m}^{-2 / 3}$ $\left(r_{0}=5 \mathrm{~cm}\right)$; different phase screens, after phase tailoring. OL, open loop.

In the numerical simulation of laser propagation in a turbulent medium different random number seed(s) can produce different turbulent media, although its spectral density for the refractive-index fluctuations (von Karman spectrum) and index structure constant are the same. It can be seen from Table 7 that the Strehl ratios and phase variances with and without an $\mathrm{AO}$ system are different for different turbulent media.

Referring to Table 2 as the turbulence strength increases, we see that the Strehl ratios with and without an AO correction rapidly decrease and that $\sim 50$ turbulence realizations are sufficient for usable average results.

There are two methods of calculating the image of the wave field on each subaperture. These are referred to as the FFT method and the integration method. The former uses the FFT to evaluate wave propagation equation (5) with $n_{1}=0$. The latter uses a direct numerical integration to evaluate Eq. (15) or its equivalent. Equation (5) can be used only under conditions that satisfy the parabolic or the Fresnel approximation. However, Eq. (15) can be used in any case. To use Eq. (5) and the FFT

Table 8. Comparison of Focusing Calculations with the FFT and the Integration Method

\begin{tabular}{cccc}
\hline Times & OL & FFT & Int. \\
\hline 1 & 0.338 & 0.819 & 0.819 \\
2 & 0.406 & 0.827 & 0.828 \\
3 & 0.311 & 0.836 & 0.836 \\
4 & 0.268 & 0.826 & 0.826 \\
5 & 0.236 & 0.828 & 0.828 \\
10 & 0.236 & 0.844 & 0.845 \\
20 & 0.221 & 0.840 & 0.840 \\
30 & 0.237 & 0.839 & 0.839 \\
40 & 0.233 & 0.834 & 0.834 \\
50 & 0.245 & 0.834 & 0.834 \\
\hline
\end{tabular}

Computational conditions: iteration times, $4 ;{C_{n}}^{2}=2.78 \times$ $10^{-14} \mathrm{~m}^{-2 / 3}\left(r_{0}=5 \mathrm{~cm}\right)$; others, see Table 7 . OL, open loop. method, the focal length of each subaperture is artificially increased. Table 8 shows that computational results with the FFT to calculate focusing of the wave field onto each subaperture agree well with those from the integration method. The computer time required with the FFT method is approximately $1 / 20$ of that with the integration method.

Real AO systems have limited response for wave-front detection, reconstruction, and correction, so the output of the compensated phase is delayed. Practically, this is the topic of dynamic control. An effective delay time $\Delta t$ is used to include this effect in the present numerical simulation of an AO system in a stationary state. The main laser beam with the compensated wave front radiates out after $\Delta t$. In this time period the phase screens move because of lateral wind and/or lateral movements of target and laser source.

Simulation results for the laser propagation and an AO system with a time delay of $4.5 \mathrm{~ms}$ with different lateral wind speeds are shown in Table 9. The results of open loop (without an AO system) and those with complete phase conjugation are included as well. It is apparent that as the wind speed increases the AO phase-compensation results worsen. The complete phase-conjugation results worsen more rapidly.

Table 9. Effects of Lateral Wind Speed $V$ on STRCC of AO Phase Compensation and the Complete Phase Conjugation

\begin{tabular}{cccc}
\hline$V(\mathrm{~m} / \mathrm{s})$ & $\mathrm{OL}$ & $\mathrm{AO}$ & $\mathrm{CPC}$ \\
\hline 0 & 0.245 & 0.834 & 0.978 \\
2 & 0.253 & 0.667 & 0.673 \\
4 & 0.254 & 0.443 & 0.412 \\
6 & 0.252 & 0.322 & 0.294 \\
8 & 0.246 & 0.266 & 0.241 \\
10 & 0.252 & 0.232 & 0.208 \\
13 & 0.250 & 0.204 & 0.182 \\
\hline
\end{tabular}

Computational conditions: delay time, $4.5 \mathrm{~ms}$; 50 turbulence realizations; others, see Table 8 .

CPC, complete phase conjugation; OL, open loop. 
If the turbulent medium does not move, the complete phase-conjugation results are better than those with AO phase compensation. However, when the wind speed is larger than a certain value (for the conditions of Table $9, V>3 \mathrm{~m} / \mathrm{s}$ ), the results with AO phase compensation become better than those with complete phase conjugation. As the lateral wind speed is increased further the complete phase-conjugation results become worse than those with no phase compensation.

It is concluded that, when the $\mathrm{AO}$ system has a certain delay time, an AO system with better phasecompensation capability does not necessarily produce a better compensation result. To obtain better AO compensation, in addition to improving the phasecompensation capability of the AO system by means of increasing the numbers of subapertures and deformable mirror actuators, it is necessary (and perhaps more important) to decrease the delay time and improve the dynamic control performance of the system.

The numerical simulation was also performed with a filtering method in which an AO system is simplified as a high-pass filter. The filtering method with a proper bandpass can give reasonable results in some cases, but it cannot give quantitative results for some other conditions even for an $\mathrm{AO}$ system in a stationary state. The filtering method is only a rough approximation.

A numerical simulation including the effects of noise, detection error, and dynamic control is in progress.

The authors gratefully acknowledge the many helpful discussions with Wenhan Jiang.

\section{References}

1. R. K. Tyson, Principles of Adaptive Optics (Academic, New York, 1991).
2. W. Jiang and H. Li, "Hartmann-Shack wavefront sensing and wavefront control algorithm," in Adaptive Optics and Optical Structures, J. J. Schulte-in-den-Baeumen and R. K. Tyson, eds., Proc. SPIE 1271, 82-93 (1990).

3. C. Boyer, V. Michon, and G. Rousset, "Adaptive optics: interaction matrix measurements and real time control algorithms for the COME-ON project," in Amplitude and Intensity Spatial Interferometry, J. B. Breckinridge, ed., Proc. SPIE 1237, 406423 (1990).

4. R. J. Noll, "Zernike polynomials and atmospheric turbulence," J. Opt. Soc. Am. 66, 207-211 (1976).

5. J. A. Fleck, Jr., J. R. Morris, and M. D. Feit, “Time-dependent propagation of high energy laser beams through the atmosphere," Appl. Phys. 10, 129-160 (1976).

6. J. M. Martin and S. M. Flatte, "Intensity images and statistics from numerical simulation of wave propagation in 3-D random media," Appl. Opt. 27, 2111-2126 (1988).

7. B. M. Welsh and C. S. Gardner, "Effects of turbulence-induced anisoplanatism on the imaging performance of adaptiveastronomical telescopes using laser guide stars," J. Opt. Soc. Am. A 8, 69-80 (1991).

8. R. R. Parenti and R. J. Sasiela, "Laser-guide-star systems for astronomical applications,” J. Opt. Soc. Am. A 11, 288-309 (1994).

9. P. B. Ulrich and L. E. Wilson, eds., Propagation of High-Energy Laser Beams through the Earth's Atmosphere, Proc. SPIE 1221, (1990); P. B. Ulrich and L. E. Wilson eds., Propagation of High-Energy Laser Beams through the Earth's Atmosphere II, Proc. SPIE 1408, (1991).

10. R. V. Digumarthi, N. G. Metha, and R. M. Blankinship, "Effects of a realistic adaptive optics system on the atmospheric propagation of a high energy laser beam," in Propagation of High-Energy Laser Beams through the Earth's Atmosphere, P. B. Ulrich and L. E. Wilson, eds., Proc. SPIE 1221, 157-165 (1990).

11. C. A. Primmerman, T. R. Price, R. A. Humphreys, B. G. Zollars, H. T. Barclay, and J. Herrman, "Atmosphericcompensation experiments in strong-scintillation conditions," Appl. Opt. 34, 2081-2088 (1995). 